\title{
Maximum Throughput in Wireless Sensor Network Using Modified Leach Protocol Using Matlab
}

\author{
*T.Vidya ${ }^{1,}$ k.vanisree ${ }^{2}$, k.Naveen ${ }^{3}$ \\ ${ }^{1}$ (M.tech student in Mlrit Hyderabad ,India), ${ }^{2}$ (professor in Engg College, Hyderabad), ${ }^{3}($,Assistant professor in \\ Mlrit ,Hyderabad, India)
}

\begin{abstract}
Wireless sensor network (WSN) technologies are increasingly employed in recent years for monitoring purposes in various fields ranging from the engineering industry to our immediate home environments due to their ability to intelligently monitor remote locations at low cost. There are various areas where research activities are going on in Wireless sensor networks. These are deployment, localization, Synchronization, Calibration, DataAggregation and Data Dissemination, Security, Middleware, Database Centric and Querying, etc One of popular area of interest is optimizing strategies of routing protocols with respect to energy utilizationof sensor nodes. Custering mechanism is one of the popular WSNs routing mechnisms, and it has proven to be an effective approach for organizing the network into a connected hierarchy In this project work we have proposed a new enhanced MODLEACH protocol after analysing the previous MODLEACH algorithm. The result shows that the network lifetime and rate of transferring packets to BS have improved.
\end{abstract}

Keywords: Modleach, sensor nodes, clustering, network lifetime, packets to BS

\section{Introduction}

A wireless sensor network (WSN) consist of hundreds to thousands of low power multi-functional sensor nodes work in an unattended environment and have sense, computation and communication ability. The basic components of a node are a sensor unit, an ADC (Analog to Digital Converter), a CPU (Central Processing Unit), a power unit and a communication unit. Sensor nodes are micro-electro-mechanical systems (MEMS) that make a computable response to a change in some physical condition related to temperature and pressure. Sensor sense or compute the physical data of the area to be monitor [1].. Sensors nodes are of very small size, use extremely low energy, are operated in high volumetric densities and can be independent and adaptive to the environment. Wireless micro-sensor networks represent a new paradigm for extracting data from the environment. Conventional systems use large, expensive macro-sensors that are often wired directly to an enduser and need to be accurately placed to obtain the data. For example, the oil industry uses large arrays of geophone sensors attached to huge cables to perform seismic exploration for oil. These sensor nodes are very expensive and require large amounts of energy for operation. The most difficult resource constraint to meet is power consumption in wireless sensor networks. The use of wireless sensor networks is increasing day by day and at the same time it faces the problem of energy constraints in terms of limited battery lifetime. As each node depends on energy for its activities, this has become a major issue in wireless sensor networks. The failure of one node can interrupt the entire system or application. Every sensing node can be in active, idle and sleep modes. In active mode, nodes consume energy when receiving or transmitting data. In idle mode the nodes consume almost the same amount of energy as in active mode. While in sleep mode, the nodes shutdown the radio to save the energy. Energy constraints end up creating computational and storage limitations that lead to a new set of architectural issues. A wireless sensor network platform must provide support for a suite of application-specific protocols that drastically reduce node size, cost, and power consumption for their target application.

In WSNs, the only source of life for the nodes is the battery. Communicating with other nodes or sensing activities consumes a lot of energy in processing the data and transmitting the collected data to the sink. In many cases (e.g. surveillance applications), it is undesirable to replace the batteries that are depleted or drained of energy [2]. Many researchers are therefore trying to find energy-aware protocols for wireless sensor networks in order to overcome such energy efficiency problems as those stated above. The memory management and the resource management in WSN is done by operating Systems specially designed for WSNs. Some of them are Tiny OS,Mantis OS,nano-Qplus,etc. Amongst them tiny OS is an open source and most popular Operating System implemented in NesC language. Now routing is the major issue in WSNs and this is under network layer issues for sending the data from sensor nodes to BS. Various routing protocols for WSNs are Sensor protocols for Information via Negotiation (SPIN), Rumor Routing, Direct Diffusion, Low Energy Adaptive Cluster Hierarchy (LEACH), Threshold Sensitive Energy Efficient Sensor network protocol (TEEN), Geographical and energy aware Routing(GEAR), Sequential Assignment Routing(SAR), etc. 
Amongst them LEACH protocol is widely used in WSN, because this protocol dissipates the energy in low level. When the battery power is drained in these devices/nodes then the network cannot be used and all the nodes spend most of the energy while transmitting the data. Therefore,to increase the lifespan of the network, each node has to do only minimal work for transmitting the data. Here all the nodes are grouped into the clusters, and in each cluster one of the nodes is assigned as a Cluster Head $(\mathrm{CH})$. $\mathrm{CH}$ collects the data from the surrounding nodes and passes it to the base station. Usually, initial assignment of $\mathrm{CH}$ is random and the role of $\mathrm{CH}$ is rotated for every fixed duration so that each node will act as a $\mathrm{CH}$ at least once in its life span. $\mathrm{LEACH}$ algorithm has two phases. They are set up phase and steady state phase. Setup phase is used to choose a $\mathrm{CH}$ and steady statephase is used to maintain the $\mathrm{CH}$ during the transmission of data.

This work focusses on analyzing the optimization strategies of routing protocols with respect to energy utilization of sensor nodes in Wireless Sensor Network (WSNs). In this project, we have considered MODLEACH protocol as reference and varied the values of the $\mathrm{p}$ to further enhance the performance of the MODLEACH protocol.

\section{Related Work}

Heinzelman, et.al [3] introduced a clustering algorithm for sensor networks, called Low Energy Adaptive Clustering Hierarchy (LEACH). LEACH forms clusters by using a distributed algorithm, where nodes make autonomous decisions without any centralized control. LEACH arranges the nodes in the network into clusters and chooses one of them as $\mathrm{CH}$. The operation of LEACH is divided into rounds. Each round begins with a setup phase when the clusters are organized, followed by a steady-state phase when data is transferred from nodes to the $\mathrm{CH}$ and then to the BS.Junayed Islam et.al [4] presented a AsLEACH (An Advanced Solar Aware Leach Protocol for Energy Efficient Routing in WSNs), a clustering based protocol which introduced the idea of sensor radio model for randomization of local CHs. It enhanced data aggregation by FIFO priority scheme and collision minimized non-persistent Carrier Sense Multiple Access (CSMA). The authors proposed a clustering routing protocol by the name of Enhanced LEACH [5], which extended LEACH protocol by balancing energy consumption in the network. Their simulation results show that Enhanced LEACH outperforms LEACH in terms of network lifetime and power consumption minimization. Meena Malik, Dr. Yudhvir Singh[6] This paper states that Wireless Sensor Network is a network of sensor nodes without having any central controller. Its growth is expeditiously increasing and that's why there is an immense field for research in this area. Sensors depend entirely on the trust of their battery for power, which cannot be revitalized or substituted. So the design of energy aware protocol is essential in respect to enhance the network lifetime. LEACH is energy efficient hierarchical based protocol that balances the energy expense, saves the node energy and hence prolongs the lifetime of the network. So this paper presents a detailed review and analysis of LEACH protocol. Comparison of various network parameters is done in the form of tables and graphs. The simulation work has been carried out by using own set of parameters and in the last of the paper conclusions is drawn. Wang and Yong [7] proposed cluster head selection by the pseudo cluster concept. Load monitor and Load leisure mechanism is used to balance the load and stability of the topology of the network. Simulation result shows that LEACH-P Protocol effectively increase energy utilization efficiency, lengthens network lifetime and balances network load. Mao Ye et al. [8] proposed EECS: An Energy Efficient Clustering Scheme for periodical data collecting applications in WSNs. During the election phase for CHS, a fixed number of candidate nodes are elected and competitor nodes are checked based on their residual energy. The competition is conducted locally and with no iteration, thus decreasing the message overhead and also helps in the even distribution of the cluster heads. To distribute the energy consumption among the sensors in the cluster formation phase a novel approach is used. EECS is fully distributed and highly energy efficient than LEACH. The simulation results proved that EECS prolonged the network lifetime as much as $135 \%$ of LEACH. In [9], authors have introduced an AdLEACH static clustering based heterogeneous routing protocol with a cluster head selection technique adopted from DEEC . It enhances both LEACH and DEEC protocols both in terms of energy efficiency and throughput. D.Mehmood et.al [10] has given a MODLEACH protocol by introducing efficient cluster head replacement scheme and dual transmitting power levels. Shou et al. [11] proposed a simulated annealing (SA) algorithm to discover the optimal solution with better position to minimize the energy loss of cluster heads. Initially, a group of $\mathrm{CHs}$ are selected by LEACH-C algorithm. For reducing the number of retransmission and considering the acknowledgment, a $\mathrm{CH}$ energy consumption model is created. This model use the quadratic sum of the distances from the $\mathrm{CH}$ to its member nodes and the largest energy consumption for a single $\mathrm{CH}$ for the next round is estimated, and all nodes that has residual energy greater than the computed energy consumption will be used for a new round of SA to give a better solution. Therefore, loss of the energy in $\mathrm{CH}$ can be minimized, to increase the lifetime of WSN. In WSN most of the communication links only exist temporally and establishing end to end connection is not possible for data delivery. Therefore routing is done based on the probability of nodal contact Ramesh and Somasundaram[12] proposed a energy efficient clustering protocol OFZ-LEACH to solve the issue by forming Far-Zone. Far-Zone is a set $\mathrm{p}$ of sensor nodes which are moving at the locations where the 
energies of those nodes are smaller than a threshold. Exponentially Weighted Moving Average (EWMA) scheme is applied for finding and updating nodal contact and based on EWMA; some functions are used for forming clusters and selection of gateway. Taneja and Bhalla [13] proposed an enhanced version of LEACH: Three Levels Hierarchical Clustering LEACH Protocol (TLHCLP) for Homogeneous WSNs.Base station is considered as the location centre and a pre defined radius is used. Nodes are categorized as nodes inside the radius and remaining nodes in the outside radius. Cluster Heads that are situated in the outside the radius discover the nearest Cluster Head which is inside the radius and forward the data to the $\mathrm{CH}$ inside the radius. Then these inside Cluster Heads aggregate the data and send it to the Base Station. The proposed TLHCLP technique is compared with the original LEACH protocol. Simulations were conducted to evaluate the performance of these two protocols and good results are obtained. Simulation results proved that TLHCLP improves network lifetime by an order of magnitude compared with LEACH. [14] introduce a routing mechanism which carries the network operation in such a way that the network lifetime is enhaced due to their energy hole removal mechanism.

\section{Proposed Protocol}

In this section at first principle of LEACH protocol is described. Next to this the proposed modification is prescribed. In clustering based LEACH protocol, includes a cluster formation technique, nodes that are selforganized. Algorithms for adapting clusters and rotating cluster head among all the nodes. In this work our proposed modified Leach is modified by replacing the existing clustering method into temporary cluster head (TCH) choosing scheme. The results show that LEACH can improve by this TCH choosing technique.

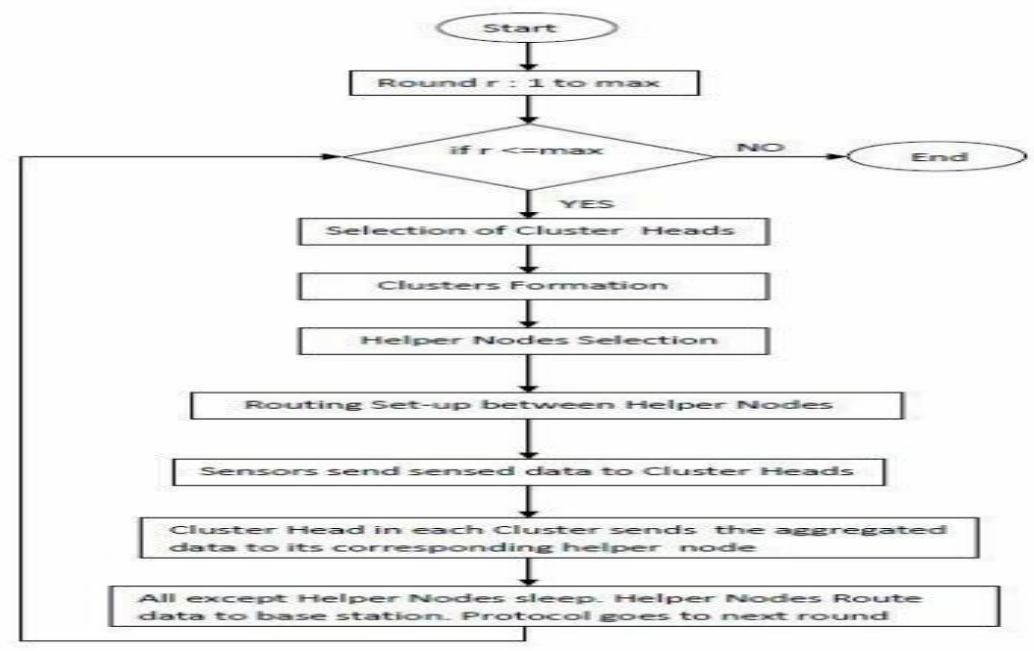

Figure1: flow chart for proposed MODLEACH Algorithm

\subsection{Cluster Head Selection}

The clusters is used for transmitting data to the base station leverages the advantages of small transmit distance for most nodes, requiring only a few nodes to transmit far distances to the base station. In our proposed protocol the classical clustering algorithms is adopted by using adaptive clusters and rotating cluster-heads, allowing the energy requirements of the system to be distributed among all the sensors. In addition, reduce the amount of data that must be transmitted to the base station. This achieves a large reduction in the energy dissipation, as computation is much cheaper than communication.

The operation of our proposed protocol is broken up into rounds, where each round begins with a setup phase, when the clusters are organized, followed by a steady-state phase, when data transfers to the base station occur. In order to minimize overhead, the steady-state phase is long compared to the set-up phase.

\subsection{Cluster-Head For The Current Round:}

Recall from LEACH model described, the cluster-heads are stochastically selected. In order to select cluster-heads each node $n$ can become a cluster-head with a probability P and every node must become clusterhead once every $1 / \mathrm{p}$ rounds. Intuitively, it means we have $n * P$ clusters and cluster-heads per round. Let the nonelected nodes be a member of set $G$ in the past 1/p.each sensors choosen a random number between 0 and 1 . If the number is less than a threshold $T(n)$, the node becomes a cluster-head for the current round. The threshold is set as follows: 
$\mathrm{T}(\mathrm{n})=\left(\mathrm{p} /\left(1-\mathrm{p}^{*} \bmod (\mathrm{r}, \operatorname{round}(1 / \mathrm{p}))\right)\right)$ then cluster head $=1$

otherwise

$$
0 \text { if, } n \in G
$$

Where $\mathrm{p}=$ the desired percentage of cluster heads (e.g., $\mathrm{p}=0: 05), \mathrm{r}=$ the current round,

and $\mathrm{G}$ is the set of nodes that have not been cluster-heads in the last $1 / \mathrm{P}$ rounds.Our proposed modleach algorithm the threshold $T(n)$, is calculated by

$\mathrm{T}(\mathrm{n})=(\mathrm{p} /(1-\mathrm{p} * \bmod (\mathrm{r}, \operatorname{round}(1 / \mathrm{p})))) * \mathrm{Q}$ then cluster head $=1$

otherwise

0 if, $n \in G$

$\mathrm{Q}$ is an additional quantity that can be defined as a function of the ratio of the residual energy of each node. to be a deterministic quotient that is computed every round for all the nodes in order to achieve improvements on the MODLEACH protocol.

\section{Simulation Environment}

The performance of new protocol was analyzed using MATLAB

\subsection{Simulation Parameters}

\begin{tabular}{|l|l|}
\hline Parameters & Value \\
\hline N no. of nodes & 100 \\
\hline P probability vectors & 0.6 \\
\hline Eo Initial energy & 0.5 \\
\hline ETX energy loss on transmission & $50 * 0.000000001$ \\
\hline ERX energy loss on receiver & $50 * 0.000000001$ \\
\hline Emp energy loss on cluster switch & $5 * 0.00000001$ \\
\hline Efs energy loss on forward & $0.0013 * 0.000000000001$ \\
\hline EDA energy loss on delay & $5 * 0.000000001$ \\
\hline maximum number of rounds & 2500 \\
\hline distance between cluster head and base station & do=sqrt(Efs/Emp); \\
\hline
\end{tabular}

\subsection{Simulation Results}

4.2.1 Dead node: Network lifetime is defined as the time difference when network is set up and time when first node died. We have calculated the network lifetime through certain parameters like Round No on whish first node died. As the nodes are increased, network lifetime is increased.

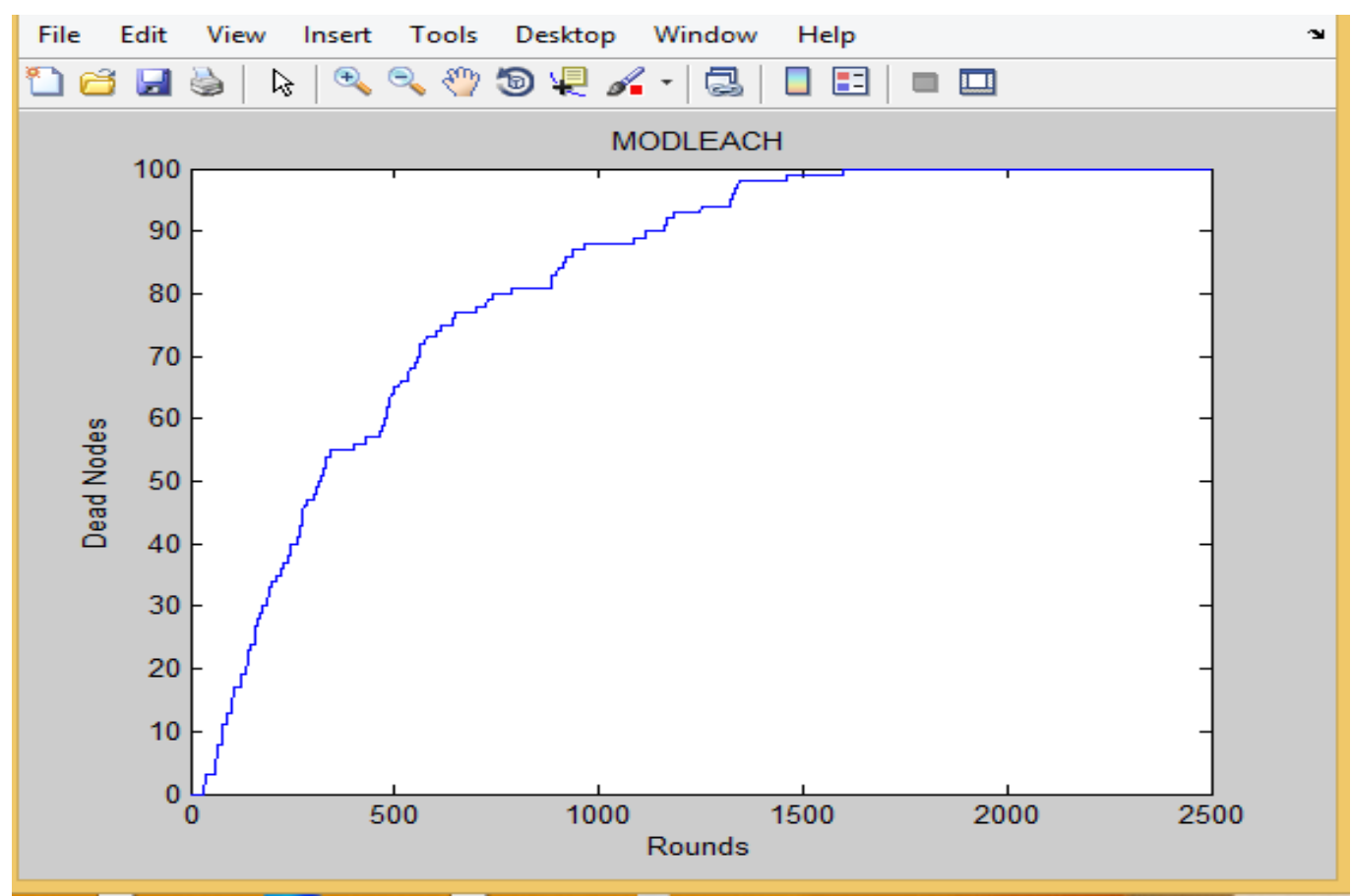

Figure4.1 Dead nodes Vs No of Rounds for MODLEACH protocol 


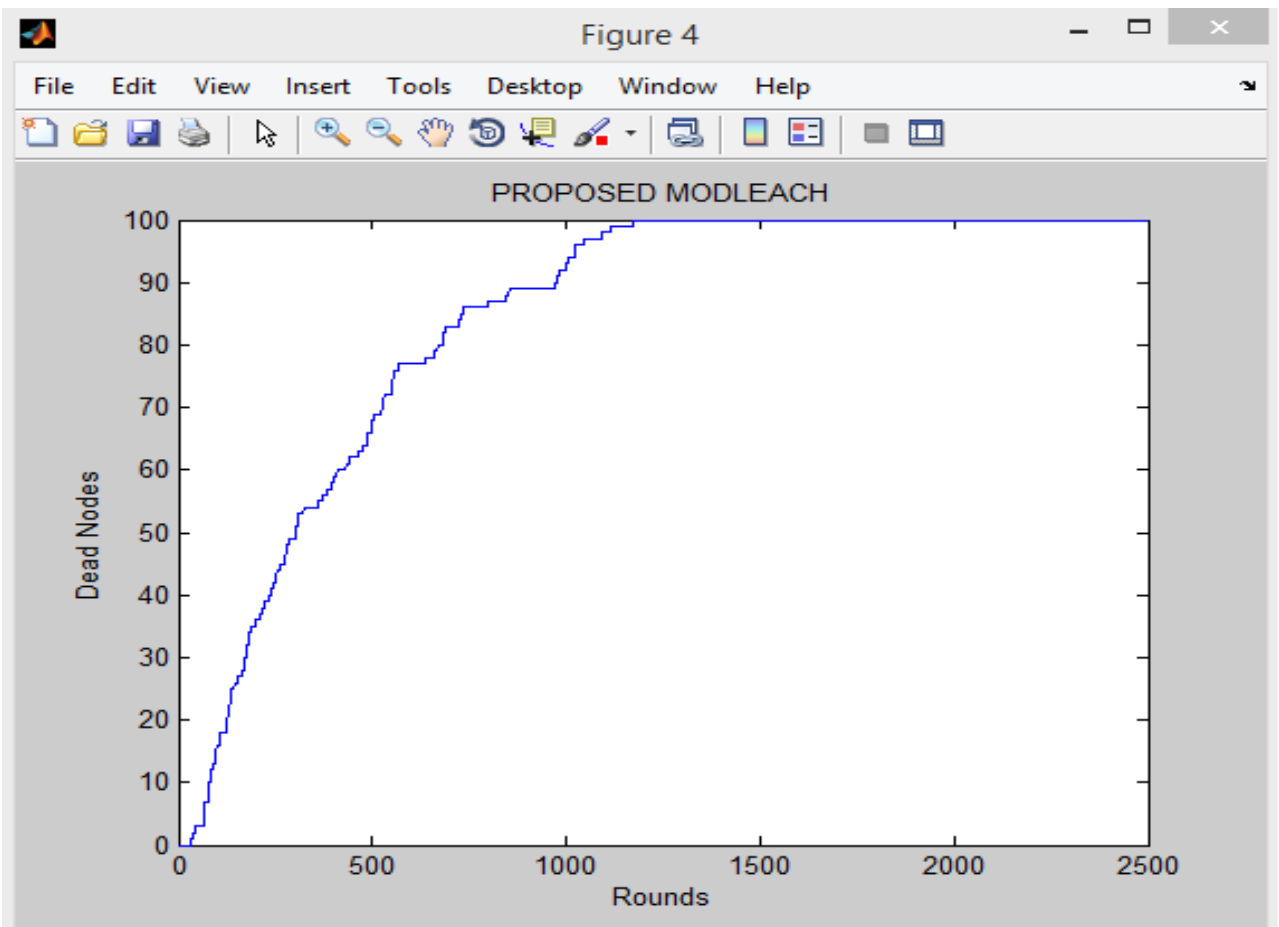

Figure4.2 Dead nodes Vs No of Rounds for proposed MODLEACH protocol

The keep analysis from the above figures 4.2 and 4.1 is the number of dead nodes is calculated into our proposed MODLEACH protocol .It shows that at 500 rounds both protocol shows approximately the number of dead nodes are same, but where as at 1000 and 1500 rounds our proposed protocol gives the number of dead nodes are more when compare with MODLEACH protocol, it means that the our proposed protocol gives more network life time.

4.2.2 Packets to BS: The amount of data received by the base station describes the rate of the accuracy of the nodes, throughput. The more data received means high accuracy. The throughput of the sensor network is measured by the total number of packets sent to base station, packets sent to cluster head during the network lifetime and cluster head formation.

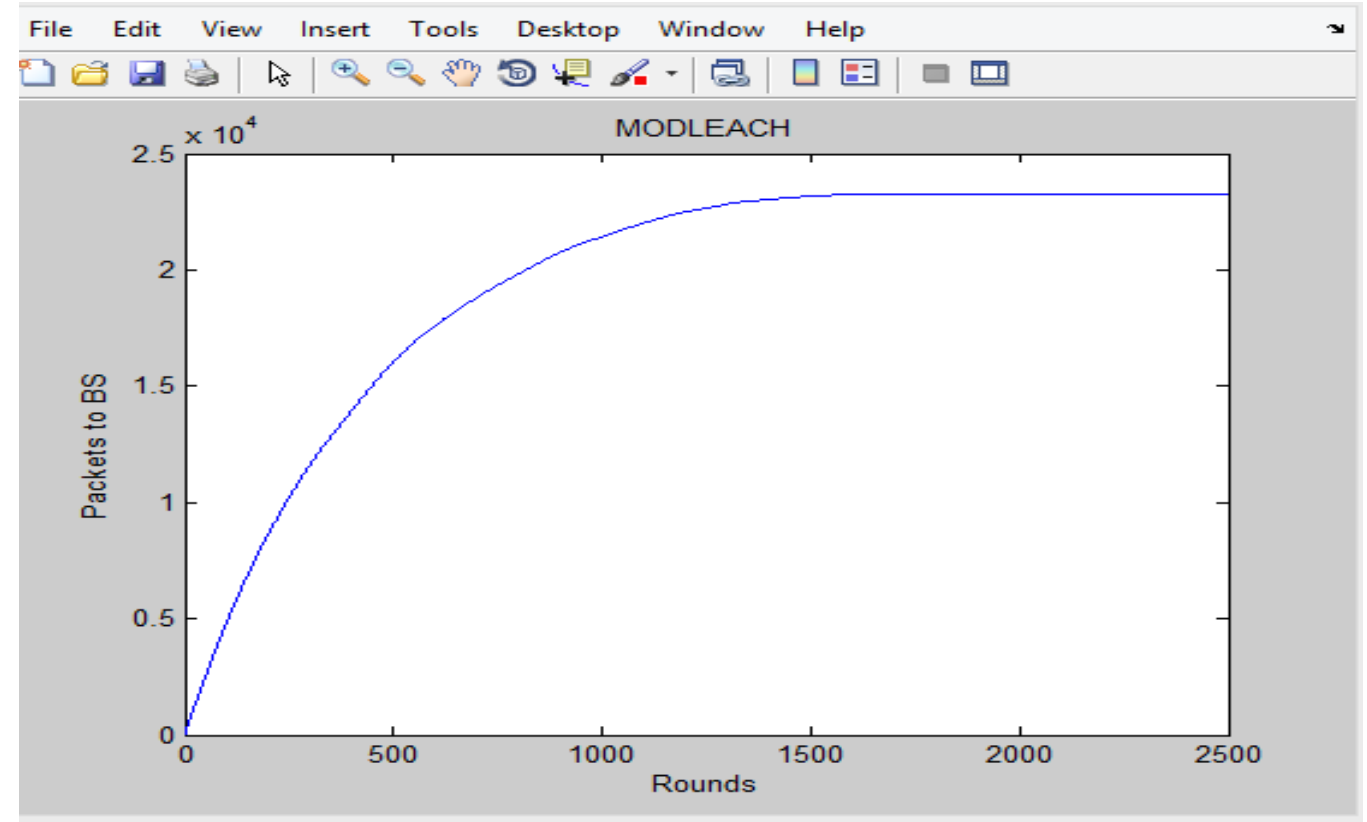

Figure4.3 Packets to BS Vs No of Rounds for MODLEACH protocol 


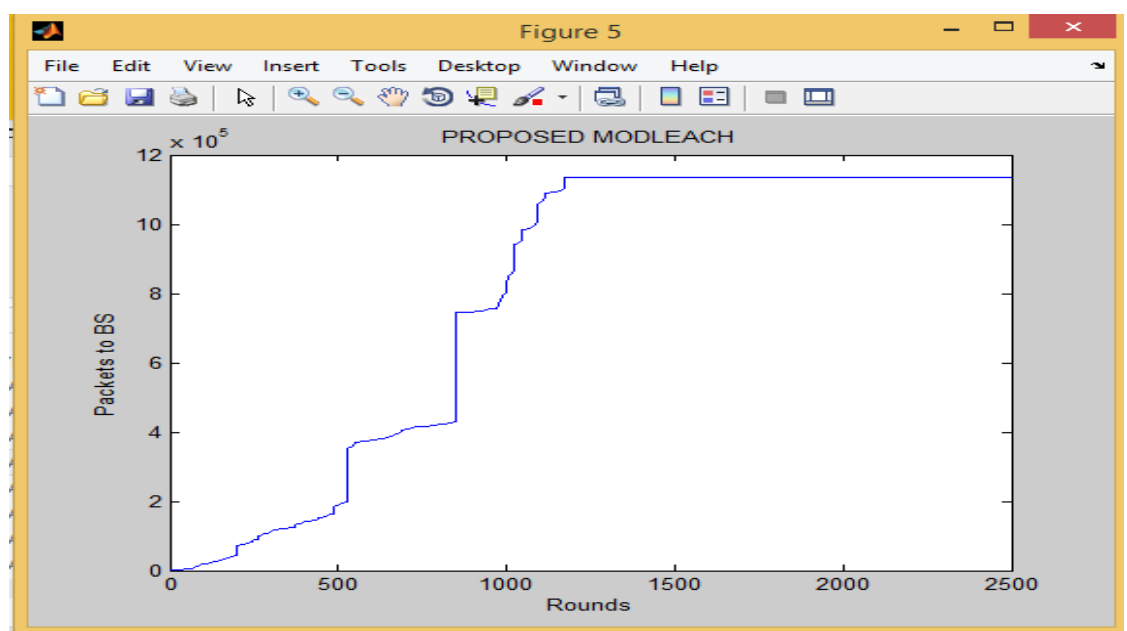

Figure4.4 Packets to BS Vs No of Rounds for proposed MODLEACH protocol

The simulation results of the figures 4.3 and 4.4 shows that our proposed MODLEACH protocol sends more packets to BS from 0- 2500 rounds, it means that our proposed protocol gives high throughput when compared with MODLEACH protocol.

Packets to CH : the packets transferred to cluster heads in various rounds of the network taking into consideration the various positions of the sink i.e. on the origin, on x-axis, on y-axis, in the middle of the network etc.

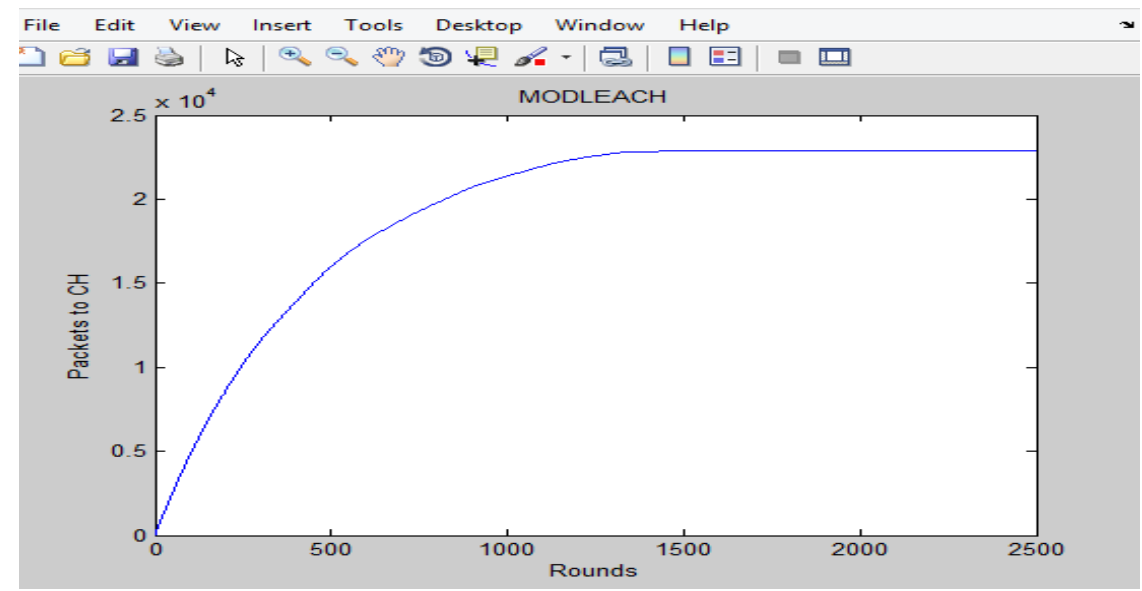

Figure4.5 Packets to $\mathrm{CH}$ Vs No of Rounds for MODLEACH protocol

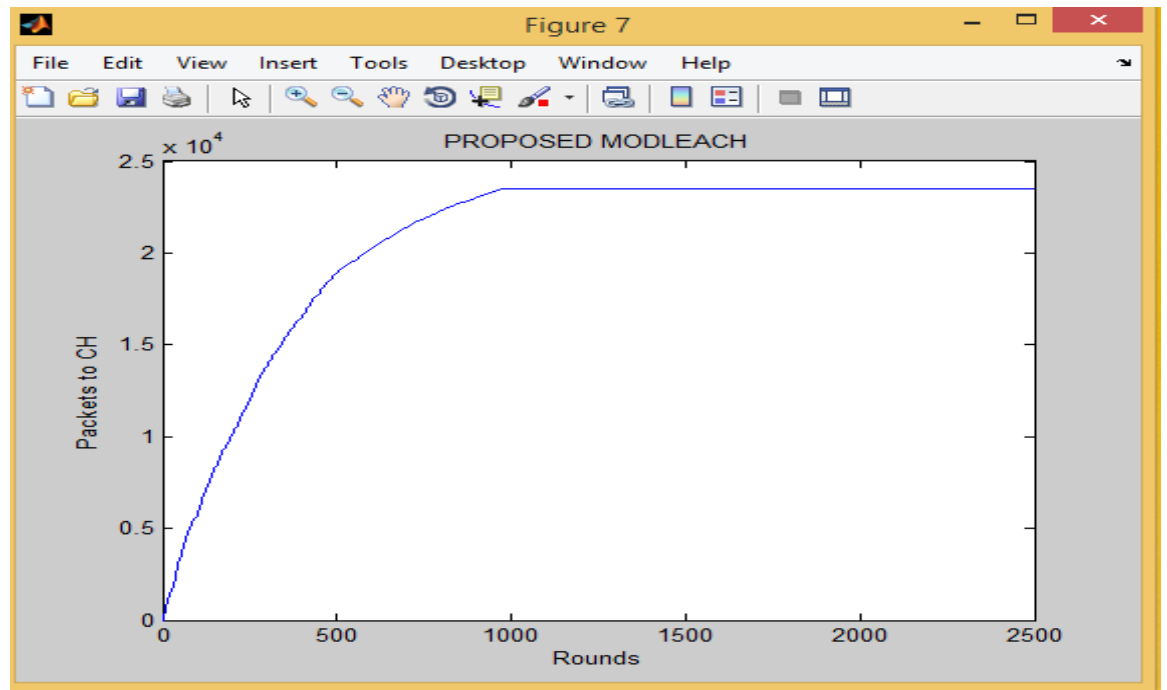

Figure4.6 Packets to CH Vs No of Rounds for proposed MODLEACH protocol 
The number of packets sent to cluster head also increases as the number of rounds increases.Also keep observation fm the above figures our proposed MODLEACH protocol sent more packets to cluster head then MODLEACH protocol.

\section{Conclusion And Future Scopes}

LEACH protocol is one of the routing protocols based on clustering algorithm to calculate the life time of the network and Throughput of the Network. A new protocol was proposed based on existing LEACH protocol to achieve high throughput and increases the network life time. Throughput was analyzed by calculating the number of packets sent to Base Station with various No. of Rounds in the network. The network life time is calculated by using No of Dead nodes by considereing Different No.of rounds .The performance analysis using MATLAB shows that the number of of packets sent to Base Station is increasing in each round than exiting algorithm. And also the No of Dead nodes are increasing ,the life time of the network is increased .Thus the new proposed MODLEACH protocol is suitable to increases the throughput and life time of the network .Further the work on fusion can be carried out in cluster selection. Hence there is need to develop the new protocol which spends least energy in receiving and fusion of the data to base station and much more efficient mechanisms for the cluster head replacement. There are several other factors like bandwidth, delay that affects the performance of the network which are correlated. But resolving the acute relationships among the parameters are difficult. Hence the work can be further improved by incorporating fuzzy decisions along with hard decision Maximization problem.

\section{Acknowledgments}

I express my sincere gratitude to my guide Ms k.vanisree for her constant help, encouragement and inspiration throughout the project work. Without her invaluable guidance, this work would never have been a successful one. I would also like to thank all the faculty members of the department for their valuable suggestions and helpful discussions. Last, but not the least, I would like to thank my husband Prakash ,my daughter jesmitha and closest friends and of course parents who have been the backbone, advisors and constant source of motivation throughout the work

\section{References}

[1]. D. Mahmood and N. Javaid et al "MODLEACH: A Variant of LEACH for WSNs", IEEE, 2013

[2]. Xu, Jia, Ning Jin, Xizhong Lou, Ting Peng, Qian Zhou, and Yanmin Chen. "Improvement of LEACH protocol for WSN.", In Fuzzy Systems and Knowledge Discovery (FSKD), IEEE 9th International Conference on, pp. 2174-2177, 2012.

[3]. W. Heinzelman, A. Chandrakasan, and H. Balakrishnan. "Energy-Efficient Communication Protocols for Wireless Microsensor Networks”. In Proceedings of Hawaiian International Conference on Systems Science, January 2000.

[4]. Junayed Islam, Muhidul Islam, Nazrul Islam "A-sLEACH: An Advanced Solar Aware Leach Protocol for Energy Efficient Routing in Wireless Sensor Networks" Department of Computer Science \&Engineering Khulna University of Engineering \& Technology Khulna- 920300, Bangladesh, 2004.

[5]. A.Koucheryavy, Ahmed Salim, and Walid Osamy, "Enhanced LEACH Protocol for Wireless SensorNetworks", St. Petersburg University of Telecommunication, 2009.

[6]. Meena Malik and Dr. Yudhvir Singh, "Energy Efficient Routing Protocols for wireless sensor network: A survey" NCACCNES, Mar2012.

[7]. Wangang Wang and Yong Peng, “LEACH Algorithm Based on Load Balancing”, TELKOMNIKA Indonesian Journal of Electrical Engineering, Vol. 11, No. 9, pp. 5329-5335, 2013.

[8]. Mao Ye, Chengfa Li, Guihai Chen and Jie Wu, "EECS: An Energy Efficient Clustering Scheme in Wireless Sensor Networks", Proceedings of IEEE International Performance, Computing and Communications Conference, pp. 535-540, 2005.

[9]. A. Iqbal, M. Akbar, N. Javaid, S.H. Bouk, M. Ilahi, R. D. Khan, "Advanced LEACH: A Static Clustering-based Heteroneous Routing Protocol for WSNs", J. Basic. Appl. Sci. Res., 3(5)864-872, 2013.

[10]. D. Mahmood, N. Javaid, S. Mehmood, S. Qureshi, A.M. Memon, T. Zaman, "MODLEACH: a variant of LEACH for WSNs"26th IEEE Canadian Conference on Electrical and Computer Engineering (CCECE2013), Regina, Saskatchewan, Canada, 2013.

[11]. Shou Shi, Xinning Liu and Xuemai Gu, "An energy-efficiency Optimized LEACH-C for wireless sensor networks", Proceedings of 7th International ICST Conference on Communications and Networking in China, pp. 487-492, 2012.

[12]. K. Ramesh and K. Somasundaram, "Optimized FZ-LEACH using Exponential Weighted Average for Wireless Sensor Networks", Advances in Computer Science and Information Technology, Second International Conference, CCSIT, Vol. 86, pp. 473-481, 2012.

[13]. Himanshu Taneja and Parvinder Bhalla, "An Improved Version of LEACH: Three Levels Hierarchical Clustering LEACH Protocol (TLHCLP) for Homogeneous WSN”, International Journal of Advanced Research in Computer and Communication Engineering, Vol. 2, No. 9, pp. 3610-3615, 2013.

[14]. M. B. Rasheed, N. Javaid, Z. A. Khan, U. Qasim and M. Ishfaq, "E-HORM: AN ENERGY EFFICIENT HOLE REMOVING MECHANISM IN WIRELESS SENSOR NETWORKS", 26th IEEE Canadian Conference on Electrical and Computer Engineering (CCECE2013), Regina, Saskatchewan, Canada, 2013.

[15]. R. Shah, J. Rabaey,Energy Aware Routing for Low Energy Ad hoc Sensor Networks, Proceedings of IEEE Wireless Communications and NetworkingConference (WCNC), 2002.

[16]. Li C-f, Cheng G-h, Ye M. et al,An uneven cluster-based routing protocol forwireless sensor networks, Chinese Journal of Computers 30(8), pages 2730,2007

[17]. Tim Nieberg, Stefan Dulman, Paul Havinga, Lode wijk v. Hoesel, Jian Wu, "Collaborative Algorithms for Communication in Wireless Sensor Networks", Kluwer Academic Publishers ISBN:1-4020-7668-1 Pages: 271 -294, 2003.

[18]. Xiaoyan Hong, Mario Gerla, Yunjung Yi, Kaixin Xu, and TaekJin Kwon, "Scalable AdHoc Routing in Large, Dense Wireless Networks UsingClustering and Landmarks," in Proc of IEEE International Conference on Communications (ICC 2002 ), April 2002. 
[19]. M. Ben Salah, A. Boulouz. "Energy Efficient Clustering Based On LEACH." IEEE, 2016: 1-3.

[20]. Tejas Rana." Performance Analysis of Clustering Protocols for Wireless Sensor Network ", International Research Journal of Engineering and Technology(IRJET),e-ISSN: 2395 -0056Volume: 04 Issue: 03| March-2017

IOSR Journal of Electronics and Communication Engineering (IOSR-JECE) is UGC approved Journal with Sl. No. 5016, Journal no. 49082.

T. Vidya. "Maximum Throughput in Wireless Sensor Network Using Modified Leach Protocol Using Matlab." IOSR Journal of Electronics and Communication Engineering (IOSR-JECE) 12.4 (2017): 01-08. 